\title{
Myxoma virus therapy for human embryonal rhabdomyosarcoma in a nude mouse model
}

\section{Veronica G Kinn \\ Valerie A Hilgenberg \\ Amy L MacNeill}

Department of Microbiology, Immunology and Pathology, College of Veterinary Medicine and Biomedical Sciences, Colorado State University, Fort Collins, CO, USA
Correspondence: Amy L MacNeill Department of Microbiology, Immunology and Pathology, College of Veterinary Medicine and Biomedical Sciences, Colorado State University, 300 West Drake Road, Fort Collins, CO 805I2, USA

Tel + I 97029751 I2

Fax + I 217297444 I

Email amy.macneill@colostate.edu
This article was published in the following Dove Press journal:

Oncolytic Virotherapy

8 August 2016

Number of times this article has been viewed

Abstract: Rhabdomyosarcoma (RMS) is a devastating tumor of young people that is difficult to cure. To determine if oncolytic virus therapy can improve outcomes in individuals with RMS, myxoma virus expressing a red fluorescent protein (MYXV-red) was evaluated for antitumoral effects using a murine model of RMS. Fluorescent protein was expressed in four RMS cell lines inoculated with MYXV-red, indicating that these cells were semipermissive to MYXV infection. MYXV-red replication and cytopathic effects were further evaluated using human embryonal RMS (CCL-136) cells. Logarithmic growth of MYXV-red and significant cell death were observed 72 hours after inoculation with MYXV. The oncolytic effects of MYXV-red were then studied in nude mice that were injected subcutaneously with CCL-136 cells to establish RMS xenografts. Once tumors measured $5 \mathrm{~mm}$ in diameter, mice were treated with multiple intratumoral injections of MXYV-red or saline. The average final tumor volume and rate of tumor growth were significantly decreased, and median survival time was significantly increased in MYXV-red-treated mice ( $P$-values $=0.0416,0.0037$, and 0.0004, respectively). Histologic sections of MYXV-red-treated tumors showed increased inflammation compared to saline-treated tumors $(P$-value $=0.0002)$. In conclusion, MXYV-red treatment of RMS tumors was successful in individual mice as it resulted in decreased tumor burden in eight of eleven mice with nearly complete tumor remission in five of eleven mice. These data hold promise that MYXV-red treatment may be beneficial for people suffering from RMS. To our knowledge, this is the first report of successful treatment of RMS tumors using an oncolytic poxvirus.

Keywords: poxvirus, myxoma virus, oncolytic virus, rhabdomyosarcoma

\section{Introduction}

Rhabdomyosarcoma (RMS) is one of the most common types of tumors in children, with an annual incidence of four cases per million people under the age of 20 years. ${ }^{1}$ RMS accounts for over half of the soft tissue sarcomas in children and is particularly difficult to treat with radiation and/or chemotherapy. While surgical removal of the tumor can be a successful means of treatment, complete amputation of the affected limb often is required. Regardless of tumor removal or other treatments, the overall survival rate for children with RMS is $\sim 60 \%{ }^{2}$ Therefore, there is a demand for highly selective strategies to improve survival rates without compromising the quality of life. ${ }^{3}$ Recent advances in the field of oncolytic viral therapeutics have showcased the potential effectiveness and safety of this treatment modality. ${ }^{4}$ This is the first study designed to determine if an oncolytic poxvirus could improve the outcome of RMS in a murine xenograft model. 
Many of the commonly studied oncolytic viruses originate from human pathogens and have some potential to replicate in noncancerous tissues, making them suboptimal for use in humans. In contrast, myxoma virus (MYXV) does not cause disease in humans or other vertebrates, with the exception of rabbits. ${ }^{5-12}$ Despite its species specificity in vivo and in nonneoplastic cells in vitro, MYXV productively infects cultured cancer cells from several animal species. ${ }^{13-17}$ Cells are considered fully permissive, semipermissive, or nonpermissive to MYXV infection based upon the cytopathic effects induced by inoculation of the cells with MYXV and the ability of virus to replicate within the cells. ${ }^{15} \mathrm{MYXV}$ replication in cancer cells is dependent upon aborted interferon signaling and increased activation of Akt in neoplastic cells. ${ }^{17,18}$ In cells that are fully permissive or semipermissive to MYXV infection, cytopathic effects are often significant, which suggests that MYXV could be an effective oncolytic agent in vivo. ${ }^{19-21}$

Oncolytic viruses are commonly genetically manipulated to increase safety, distinguish them from wild-type virus, enable easy detection, and/or enhance immunogenicity in vivo. The biological characteristics of recombinant MYXV that expresses the fluorescent protein marker tandem-dimer tomato (MYXV-red; previously designated vMyx-tdTr) have been extensively evaluated and are nearly identical to wild-type MYXV. ${ }^{22}$ Note that MYXV encodes several antiapoptotic proteins that delay cytopathic effects in cultured cells. ${ }^{23-25}$ These genes can be deleted from the virus to enhance apoptosis; however, this may not allow for maximal viral replication in vivo. ${ }^{13,25}$ In rodent cancer models, recombinant MYXV treatment eliminated some glioma xenografts and reduced tumor burden in several types of xenografts and allografts. ${ }^{19-21,26-29}$ Based upon these data, we chose to evaluate the oncolytic potential of MYXV-red in a murine xenograft model of RMS.

\section{Materials and methods Cells}

Human RMS (RMS13 and CCL-136) cells were purchased from the American Type Culture Collection (Manassas, VA, USA). Murine RMS (U21075 and U21089) cells were obtained from Dr Charles Keller (Children's Cancer Therapy Development Institute, Beaverton, OR, USA). Rabbit kidney epithelial (RK13) cells were a gift from Dr Richard Moyer (University of Florida, Gainesville, FL, USA). Cell lines were screened and tested negative for ectomelia virus, epizootic diarrhea of infant mice virus, lymphocytic choriomeningitis virus, lactate dehydrogenase elevating virus, mouse hepatitis virus, mouse norovirus, mouse parvovirus, minute virus of mice, polyoma virus, pneumonia virus of mice, reovirus type 3 , sendai virus, Theiler's murine encephalomyelitis virus strain GDVII, and Mycoplasma sp. by polymerase chain reaction (PCR). Noncancerous fibrocytes were isolated from a subcutaneous mass in a dog that was biopsied and diagnosed as hyperplastic mesenchymal tissue. Fibrocytes were used as a control cell type expected to be nonpermissive to MYXV infection. Cells were maintained at $37^{\circ} \mathrm{C}, 5 \%$ carbon dioxide, and $100 \%$ humidity in minimum essential medium with Earle's salts and $2.0 \mathrm{mM} \mathrm{L-glutamine} \mathrm{supplemented} \mathrm{with} 2$ $\mathrm{mM}$ L-glutamine, $50 \mathrm{U} / \mathrm{mL}$ penicillin $\mathrm{G}, 50 \mu \mathrm{g} / \mathrm{mL}$ streptomycin, $1 \mathrm{mM}$ sodium pyruvate, $0.1 \mathrm{mM}$ nonessential amino acids, and 10\% defined fetal bovine serum (FBS). Media that contained all supplements except FBS is designated as MEM-C in this paper. Sodium pyruvate and nonessential amino acids were from Corning Incorporated (Corning, NY, USA). All other cell culture reagents were from GE Healthcare UK Ltd (Little Chalfont, UK).

\section{Virus}

Dr Grant McFadden (University of Florida, Gainesville, FL, USA) provided the recombinant virus stock used in this study. Construction and characterization of myxoma virus expressing the fluorescent protein tandem-dimer tomato (MYXV-red; originally designated vMyx-tdTr) was previously described. ${ }^{22}$ MYXV-red stock was amplified in RK13 cells and cleared of cellular debris by sucrose-pad purification. Three separate preparations of virus were made and diluted in separate volumes of phosphate-buffered saline (PBS). The virus titer and volume in each preparation were appropriate for treatment injections containing $10^{6}$ plaqueforming units (pfu) of virus in $100 \mu \mathrm{L}$ of PBS for six mice. One of the preparations, designated MYXV-red*, caused an adverse respiratory reaction that necessitated euthanasia in five mice and was not used in any other mice in this study (data are shown in Figure S1 and Table S1).

\section{Cytopathic effects in cell cultures inoculated with virus}

Human (CCL-136 and RMS13) and murine (U21089 and U21075) RMS cellular monolayers were incubated at $37^{\circ} \mathrm{C}$ with one infectious MYXV-red particle per ten cells (multiplicity of infection [moi] $=0.1$ ) for 1 hour in MEM-C. The virus inoculum was then replaced by MEM-C with $10 \%$ FBS. Cells were evaluated microscopically for cytopathic effects at 24, 48, and 72 hours postinoculation as an initial test of permissivity of cells for virus infection. RK13 cells, which 
are permissive for MYXV-red infection and replication, were included as a positive control.

\section{Virus replication in CCL- 136 cells}

RK13 and CCL-136 cell monolayers were incubated with MYXV-red ( $\mathrm{moi}=0.1$ ) in $35 \mathrm{~mm}$ diameter plates and incubated at $37^{\circ} \mathrm{C}$ in $5 \% \mathrm{CO}_{2}$ for 1 hour, with rocking. The virus inoculum was removed and cells were washed three times with PBS. One and one-half milliliters of media with 5\% FBS was added to the plates. The first time point ( 0 hour) was harvested by scraping cells into the media with a rubber stopper, centrifuging the sample at $400 \times g$ for 10 minutes, resuspending the cell pellet in $1 \mathrm{~mL}$ MEM-C, and then storing the sample at $-80^{\circ} \mathrm{C}$. The remaining samples were incubated at $37^{\circ} \mathrm{C}$ in $5 \% \mathrm{CO}_{2}$. At 12 , 24,48 , and 72 hours postinoculation, cells were collected as described for the 0 -hour time point. Once all time points were collected, cells were lysed by repeated freezing then thawing, and sonicated to release viral particles. Cell lysates were serially diluted and inoculated onto uninfected RK13 cell monolayers for 1 hour at $37^{\circ} \mathrm{C}$. After inoculation, cell monolayers were washed with $\mathrm{PBS}$ repeatedly and then an overlay of equal parts of $1 \%$ agar and $2 \times$ MEM-C was added to the cell culture wells. Seven days later, the numbers of small white foci (viral plaques) were counted and viral $\mathrm{pfu} / \mathrm{mL}$ of lysate was calculated. The experiment was performed in triplicate and the average viral titer was determined at each time point to create multistep viral growth curves for RK13 and CCL-136 cells.

\section{Cell viability assays}

RK13 and CCL-136 cell monolayers were incubated with MYXV (moi =1) in triplicate as previously described. At 72 hours postinoculation, cell viability was assessed using a CellTiter-Blue ${ }^{\circledR}$ assay (Promega Corporation, Fitchburg, WI, USA) as directed by the manufacturer. Briefly, CellTiterBlue ${ }^{\circledR}$ reagent was added to each cell culture well 2 hours before analysis. Analysis was performed using a BioTek Synergy H1 Multi-Mode Reader (BioTek Instruments, Inc., Winooski, VT, USA) at $560 \mathrm{~nm}$ excitation and $590 \mathrm{~nm}$ emission wavelengths. Using this protocol, viable cells emit fluorescence at $590 \mathrm{~nm}$. Data were analyzed with an unpaired parametric $t$-test for independent samples with Welch's correction using GraphPad Prism ${ }^{\circledR} 6.04$ software (GraphPad Software, Inc., La Jolla, CA, USA).

\section{Mice}

All animal procedures were approved by an Institutional Animal Care and Use Committee (protocol \#14-5211A). Mice were treated humanely and housed according to American Association for Laboratory Animal Science guidelines and were euthanized humanely following the American Veterinary Medical Association guidelines. Four- to six-week-old, female B6.Cg-Foxn1nu/J mice (The Jackson Laboratory, Bar Harbor, ME, USA) were inoculated subcutaneously in the rear flank with $10^{7}$ CCL-136 cells in $100 \mu \mathrm{L}$ PBS. This protocol results in measurable tumor growth in $100 \%$ of nude mice $\sim 3$ months after inoculation..$^{30}$ Intratumoral injections were started once tumor length measured $5 \mathrm{~mm}$, which was designated as day 0 . On days $0,3,6$, and 13 , then every 2 weeks until euthanasia or tumor regression, eleven mice were injected with $10^{6}$ pfu of sucrose pad-purified MYXV-red diluted in $100 \mu \mathrm{L}$ PBS. Ten mice, injected with $100 \mu \mathrm{L}$ PBS on the same treatment schedule, were used as controls. Mice were euthanized by intraperitoneal injection of pentobarital (100-150 $\mathrm{mg} / \mathrm{kg}$ ) if tumor volume was calculated to be $>3.0 \mathrm{~cm}^{3}$, if the mass became ulcerated, or if mice lived $>90$ days after the first treatment injection. The number of treatments received by PBS and MYXV-red mice was compared using a Mann-WhitneyWilcoxon test for nonparametric data. GraphPad Prism ${ }^{\circledR} 6.04$ software was used for statistical comparisons.

\section{Evaluation of clinical outcomes in mice}

Mice were monitored daily. Physical appearance, weight, and tumor dimensions were recorded. Tumor length and width were measured using calipers. Tumor volumes were determined using the following formula: $0.4 \times$ length $\times$ width $^{2} .{ }^{31}$ The overall rate of tumor growth $\left(\mathrm{mm}^{3} /\right.$ day) was estimated using Microsoft Excel Software (Microsoft Corporation, Redmond, WA, USA) to calculate the slope of the best fit line between tumor volume at day 0 and euthanasia. Tumor volume and growth rate data from MYXV-red-treated and PBS-treated mice were compared using a Mann-WhitneyWilcoxon test for nonparametric data. Survival data were compared using a log-rank (Mantel-Cox) test. GraphPad Prism $^{\circledR} 6.04$ software was used for all statistical comparisons.

\section{Tissue collection}

Mice were euthanized by intraperitoneal injection of pentobarital (100-150 mg/kg) and a necropsy was performed. Internal organs were grossly examined for metastases, evidence of viral infection, or other pathology. Sections of all major organs and tumors were collected and placed in 10\% buffered formalin for histological examination by light microscopy. Other sections of each organ and tumor were stored at $-80^{\circ} \mathrm{C}$ to evaluate viral titer and DNA within the tissues.

\section{Histopathology}

Tissues collected during routine necropsy and fixed in 10\% buffered formalin were trimmed, paraffinized, sectioned, 
stained with hematoxylin and eosin, and evaluated using light microscopy. Organs were examined closely for any pathology. Lymphoid hyperplasia in lymph nodes, spleen, and/or lymphoid tissue associated with internal organs was recorded as 0 (no hyperplasia) or 1 (hyperplasia). Similarly, inflammatory cell infiltration into the tumor area was recorded as 0 (inflammatory cells absent) or 1 (inflammatory cells present). Tumor necrosis was scored to reflect the severity of the finding as a percentage of the tumor area: 0 , no necrosis; $1,1 \%-25 \%$ necrosis; 2, 26\%-50\% necrosis; 3, 51\%-75\% necrosis; 4, 76\%-100\% necrosis. Results from PBS- and MYXV-redtreated mice were compared using Man-Whitney-Wilcoxon test for nonparametric data. GraphPad Prism ${ }^{\circledR} 6.04$ software was used for all statistical comparisons.

\section{Isolation of infectious virus particles and viral DNA from tissues}

Frozen sections of internal organs and tumors from mice were individually weighed and homogenized in $2 \mathrm{~mL}$ MEM-C. Tissue homogenates were serially diluted and plaqued onto RK13 cells to determine virus titer. Briefly, homogenates were serially diluted and inoculated onto uninfected RK13 cell monolayers for 1 hour at $37^{\circ} \mathrm{C}$. After inoculation, cell monolayers were washed with $\mathrm{PBS}$ repeatedly and then an overlay of equal parts of $1 \%$ agar and $2 \times$ MEM-C was added to the cell culture wells. Seven days later, the numbers of small white foci (viral plaques) were counted and viral pfu/g of tissue was calculated. A Qiagen DNeasy Blood \& Tissue Kit (Qiagen NV, Venlo, the Netherlands) was used to extract DNA from tissue homogenates as directed. The concentration and relative purity of DNA in each sample were determined using a BioTek Take3 Micro-Volume Plate and the BioTek Synergy H1 Multi-Mode Reader. One hundred nanograms of the DNA sample was analyzed by PCR using three sets of primers to detect MYXV-red DNA within the tissues. Primer sets and specific PCR parameters are listed in Table 1.

\section{Results}

\section{Cytopathic effects are observed in RMS cell lines following MYXV-red infection}

To assess whether RMS cell lines were permissive for MYXV-red infection, cellular monolayers were incubated with MYXV-red (moi =0.1) and evaluated using red fluorescence microscopy to detect viral entry and recombinant protein expression. It was observed that a lower percentage of RMS cells expressed red fluorescent protein (which is indicative of MYXV-red entry) compared to RK13 cells in which virtually all cells were infected. Figure $1 \mathrm{~A}$ depicts fluorescent protein expression by MYXV-red at 48 hours postinoculation ( $\mathrm{moi}=0.1$ ) in fully permissive RK13 cells, CCL-136 cells, and in noncancerous mesenchymal cells from a dog (fibrocytes). As expected, $\sim 100 \%$ of the fully permissive RK13 cells contained fluorescent protein. CCL-136 cells were deemed semipermissive to MYXVred infection based upon the observance that a percentage of cells expressed fluorescent protein. Fibrocytes were confirmed to be nonpermissive to MYXV-red infection since fluorescent protein was not detected in virtually any of these cells. The amount of fluorescent protein expression observed after inoculation of MYXV-red into other RMS cell lines (RMS13, U21089, and U21075 cells) was similar to CCL-136 cells (Figure S2). At 72 hours postMYXV-red inoculation, a marked increase in the number of detached, rounded RK13 cells could be detected by phase-contrast microscopy and all cells fluoresced. A similar trend was observed in infected murine RMS cells (U21075 and U21089), but there were fewer detached, rounded cells in CCL-136 and RMS13 cellular monolayers (data not shown). These data suggest that all four RMS cell lines are semipermissive for virus infection. The effects of MYXV-red treatment on RMS cells were further evaluated using CCL-136 cells because subcutaneous injection of CCL-136 cells has been demonstrated to reliably induce RMS tumors in nude mice. ${ }^{30}$

Table I Primer sequences and specific PCR parameters used to detect MYXV-red DNA in tissues

\begin{tabular}{|c|c|c|c|c|}
\hline $\begin{array}{l}\text { Target gene } \\
\text { segment(s) }\end{array}$ & $\begin{array}{l}\text { Forward primer } \\
\text { sequence }\end{array}$ & $\begin{array}{l}\text { Reverse primer } \\
\text { sequence }\end{array}$ & $\begin{array}{l}\text { Annealing temperature } \\
\left({ }^{\circ} \mathrm{C}\right)\end{array}$ & $\begin{array}{l}\text { Product length } \\
\text { (base pairs) }\end{array}$ \\
\hline $\begin{array}{l}\text { MYXV M034L } \\
\text { (DNA polymerase) }\end{array}$ & $\begin{array}{l}5^{\prime} \text { CTG CGT AGA CGA CGG } \\
C 3^{\prime}\end{array}$ & 5' CGG AGG ATT TGG TGT CGG 3' & 60 & 600 \\
\hline $\begin{array}{l}\text { MYXV M033R and } \\
\text { M034L }\end{array}$ & $\begin{array}{l}5^{\prime} \text { CAC CCT CTT TAG TAA } \\
\text { AGT ATA CAC C } 3^{\prime}\end{array}$ & 5' GAA ATG TTG TCG GAC GGG 3' & 56 & 800 \\
\hline $\begin{array}{l}\text { MYXV MI5IR } \\
\text { (serp2) }\end{array}$ & $\begin{array}{l}5^{\prime} \text { GGC GAC TGG AGA CTG } \\
\text { G } 3^{\prime}\end{array}$ & $5^{\prime}$ CCG CGT ACG GGG GAC TG 3' & 60 & 950 \\
\hline
\end{tabular}

Note: Three partial gene segments were amplified for each tissue sample.

Abbreviations: PCR, polymerase chain reaction; MYXV, myxoma virus; MYXV-red, MYXV with red fluorescent protein. 


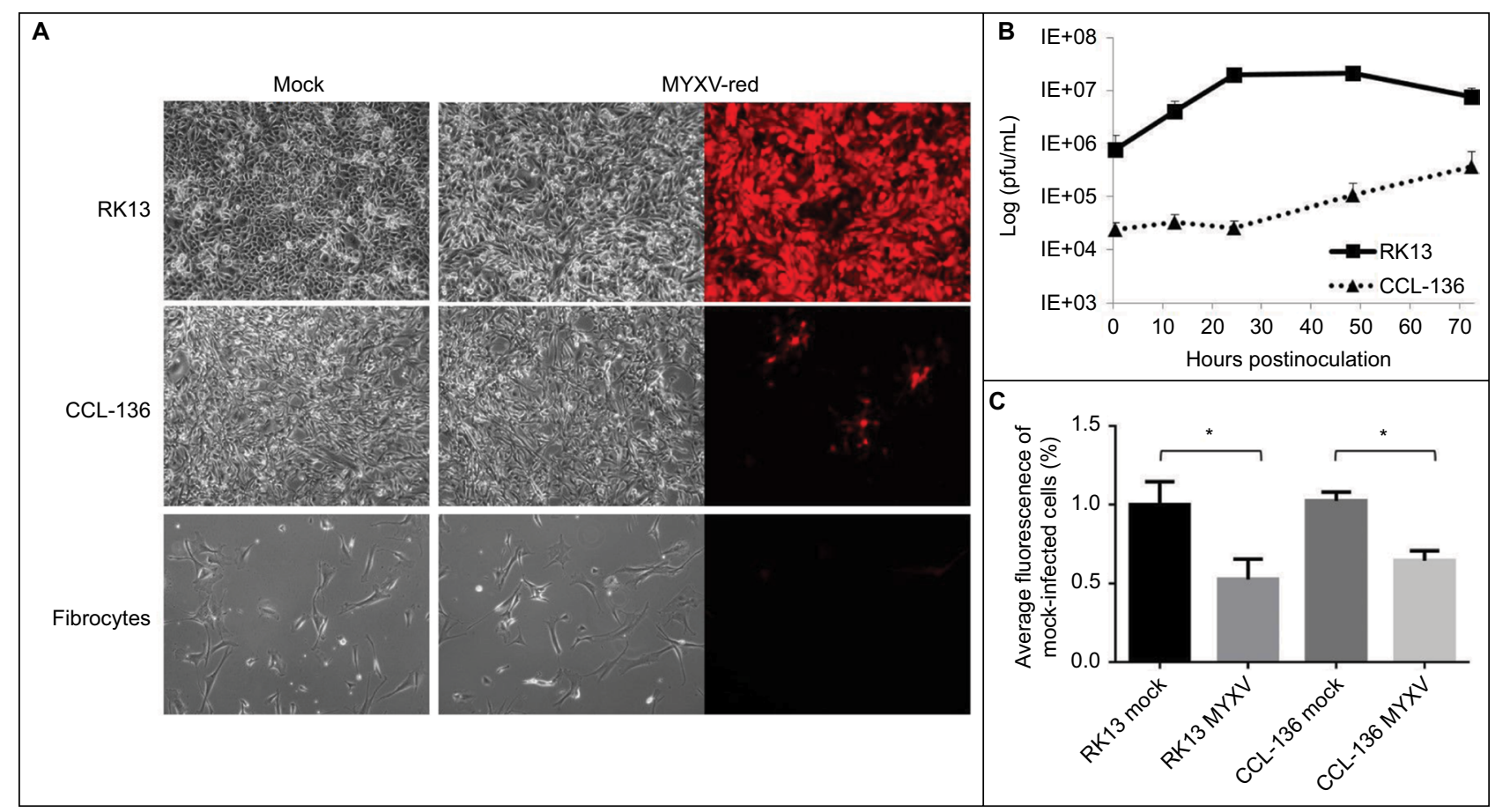

Figure I Effects of incubating cell cultures with MYXV-red ( $\mathrm{moi}=0 . \mathrm{I})$.

Notes: (A) Cytopathic effects: forty-eight hours after inoculation with MYXV-red, red fluorescence is observed in nearly all RKI3 (fully permissive) cells and in lower numbers of CCL-136 cells, but not in fibrocytes (nonpermissive) cells. Magnification I000x. (B) Viral growth curves: MYXV-red multistep growth kinetics in RKI 3 and CCL136 cells is shown. Data are expressed as the logarithm of viral plaque-forming units per milliliter (pfu/mL) of infected cell lysate over time and depict the average of three experiments. Error bars indicate standard error of the mean. (C) Cell viability: viability of RKI3 and CCL-I36 cells was determined 72 hours postinoculation with MYXV $(\mathrm{moi}=\mathrm{I})$. Data are presented as the percentage of the average fluorescence intensity of mock-infected cells. Error bars indicate standard deviation $(\mathrm{n}=6)$. $* P$-values of mock versus MYXV-infected cells $\leq 0.0004$.

Abbreviations: MYXV-red, myxoma virus with red fluorescent protein; moi, multiplicity of infection; RK I3, rabbit kidney epithelial cells; CCL-I36, human rhabdomycosarcoma cells.

\section{MYXV-red replicates in CCL-I36 cells}

Viral plaque assays were performed to construct a multistep viral growth curve and assess whether a representative human RMS cell line (CCL-136) could support the replication of MYXV-red. Cellular monolayers were incubated with MYXVred (moi $=0.1$ ), collected at several time points postinoculation, and titered onto permissive, RK13 cells. The average viral titer isolated from MYXV-red-inoculated CCL-136 cells at each time point was lower than that isolated from the permissive RK13 cells. In part, this may be due to the fact that the number of cells that are present in each cell culture well differs slightly between the cell lines, but for RK13 and CCL-136 cells this difference is minimal. More importantly, three PBS washes are performed after the virus is incubated with the cells for 1 hour to remove any virus that has not entered into the cells. By convention, this time point is defined as the zero time point on a poxvirus growth curve. Less virus was recovered from the wells containing CCL-136 cells at each time point, which reflects the fact that the virus is significantly more effective at entering into RK13 (fully permissive cells) than CCL-136 cells. Nevertheless, logarithmic growth of virus did occur in CCL-136 cells between 24 and 72 hours postinoculation, indicating that MYXV-red replicates in CCL-136 cells (Figure 1B). These findings support the conclusion that CCL136 cells are semipermissive to MYXV-red infection.

\section{MYXV induces cell death in infected CCL-I 36 cells}

Oncolysis is a desired effect of virotherapy for cancer. To determine if MYXV-red induced cell death in CCL-136 cells, cell monolayers were infected with MYXV-red $(\mathrm{moi}=1)$ and cell viability was detected by conversion of resazurin to resorufin and subsequent release of fluorescence in live cells. As seen in permissive RK13 cells, a significant decrease in fluorescent units was observed in CCL-136 cells 72 hours postinoculation with MYXV-red compared to mock-infected CCL-136 cells incubated with MEM-C only ( $P$-value 0.0004$)$. This indicates that MYXV-red induces cell death in CCL-136 cells (Figure 1C).

\section{Intratumoral injections of MYXV-red decreases tumor burden in a RMS xenograft model}

The effects of MYXV-red treatment were evaluated in mice with established RMS tumors. Multiple intratumoral 
injections of MYXV-red or PBS were administered to provide continued antigenic stimulation by the virus (Figure 2A). There was no statistical difference between the number of PBS and MYXV-red treatments administered to the two groups of mice (Table 2). During treatment, none of the mice became lethargic or stopped grooming, eating, urinating, or defecating at any time point. All mice gained weight at a rate expected for their age group based upon body weight information provided by The Jackson Laboratory (data not shown). The average end point tumor volume was 51\% smaller (Figure 2B) and overall tumor growth rate was 32\% slower (Figure 2C) in MYXV-red-treated mice $(n=11)$ compared to PBS-treated mice $(n=10)$. Nearly complete tumor regression was observed in five of the eleven MYXV-red-treated mice. The median survival time of MYXV-red-treated mice was significantly longer than PBS-treated mice (Figure 2D). These findings indicate that the virus has a desired antitumor effect.

\section{Tumor inflammation and lymphoid hyperplasia were increased in MYXV-red- treated mice}

At the time of euthanasia, no abnormalities were present in histological sections of the brain, heart, liver, pancreas, kidneys, urinary bladder, ovaries, or uterus from any of the mice. Hyperplastic lymphoid tissue was noted in five of eleven MYXV-red-treated mice; all five had mild lymph node hyperplasia, three also had enlarged lymphoid nodules in the lung, two had splenic lymphoid hyperplasia, and one had large Peyer's patches in the gastrointestinal tract. Tumor growth was markedly reduced in three of the five mice with lymphoid hyperplasia. Three of the five mice with immeasurable tumors at euthanasia had a moderate to marked histiocytic infiltrate within lymph nodes. None of the PBS-treated mice had evidence of lymphoid hyperplasia or histiocytic inflammation.

Histological analyses of tumor sections from PBS-treated mice revealed a large, discrete, round or lobulated, subcutaneous, cancerous mass. The neoplastic cells that comprised the mass were spindloid with a high nuclear to cytoplasmic ratio (Figure 3A). Anisokaryosis and anisocytosis were marked. Nuclei were oval or irregularly shaped with an open, stippled chromatin pattern and often one or more prominent, round nucleoli. Several multinucleated cells were noted. Zero to six mitotic figures were observed per field (400× magnification). Necrosis was present at the center of the tumors from eight of ten PBS-treated mice, but mild inflammation was observed only in two of ten tumors and was associated with areas of necrosis. Tumor histology from six of eleven mice

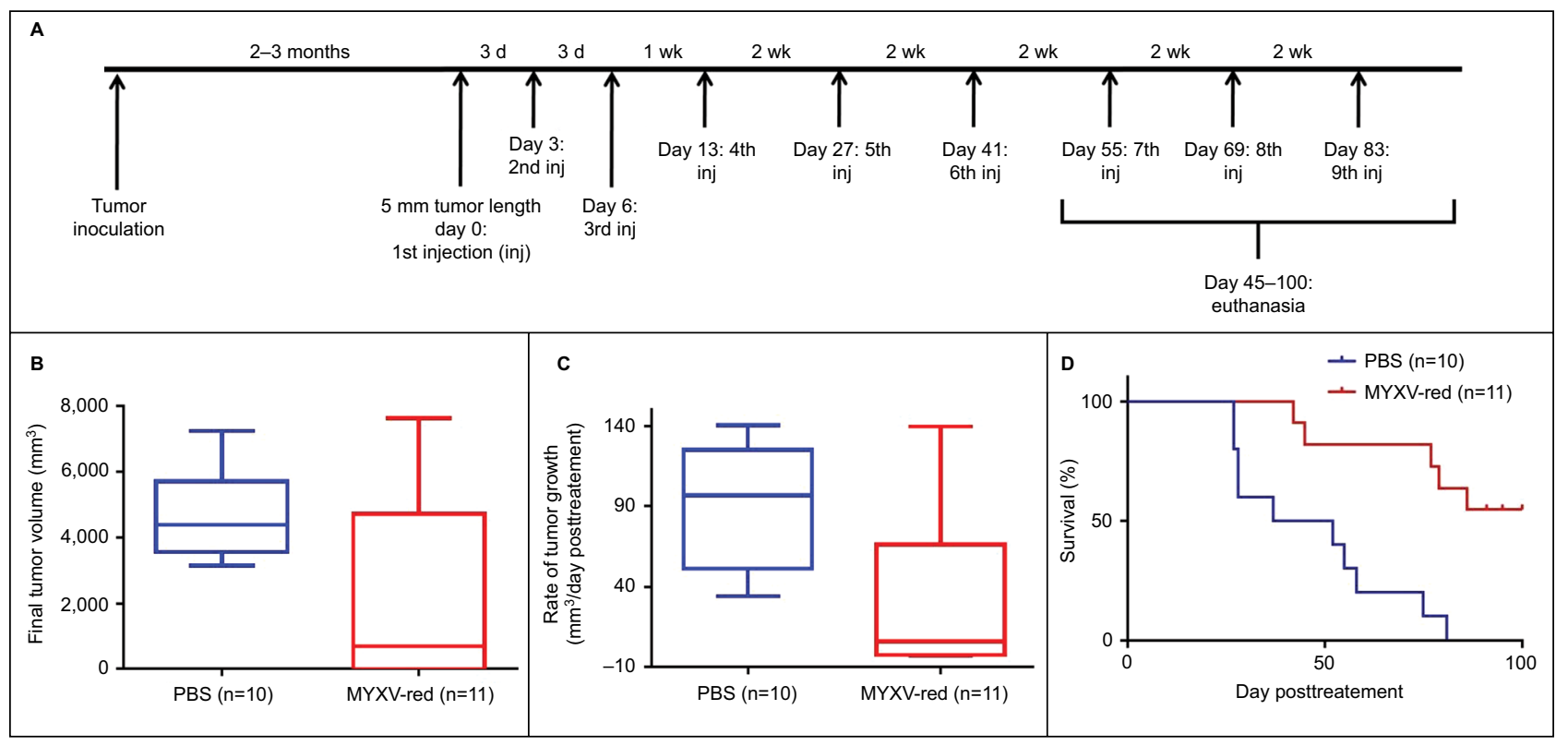

Figure 2 Effects of MYXV treatment.

Notes: (A) Treatment schedule: nude mice were inoculated subcutaneously with $10^{7} \mathrm{CCL}-136$ rhabdomyosarcoma cells. Once tumors measured 5 mm in length, injections of either $10^{6}$ plaque-forming units (pfu) MYXV-red or PBS were administered into the tumor at the indicated time points. (B) Final tumor volume: tumor volume $(0.4 \times$ length $\times$ width $^{2}$ ) was calculated at the time of euthanasia after the initial intratumoral injection of PBS or $10^{6}$ pfu MYXV-red. Median final tumor volume was significantly decreased in MYXV-red-treated mice $(P$-value $=0.0416)$. (C) Tumor growth rate: the rate of tumor growth in each mouse between the initial intratumoral injection of PBS or $10^{6}$ pfu MYXV-red and the time of euthanasia was calculated. The median tumor growth rate was significantly decreased in MYXV-red-treated mice $(P$-value $=0.0037)$. (D) Kaplan-Meier survival curves: survival times of mice treated with PBS or MYXV-red are shown. Median survival time was significantly longer in MYXV-red-treated mice $(P$-value $=0.0004)$.

Abbreviations: d, day; wk, week; inj, injection; PBS, phosphate-buffered saline; MYXV-red, myxoma virus with red fluorescent protein; CCL-I36, human rhabdomycosarcoma cells. 


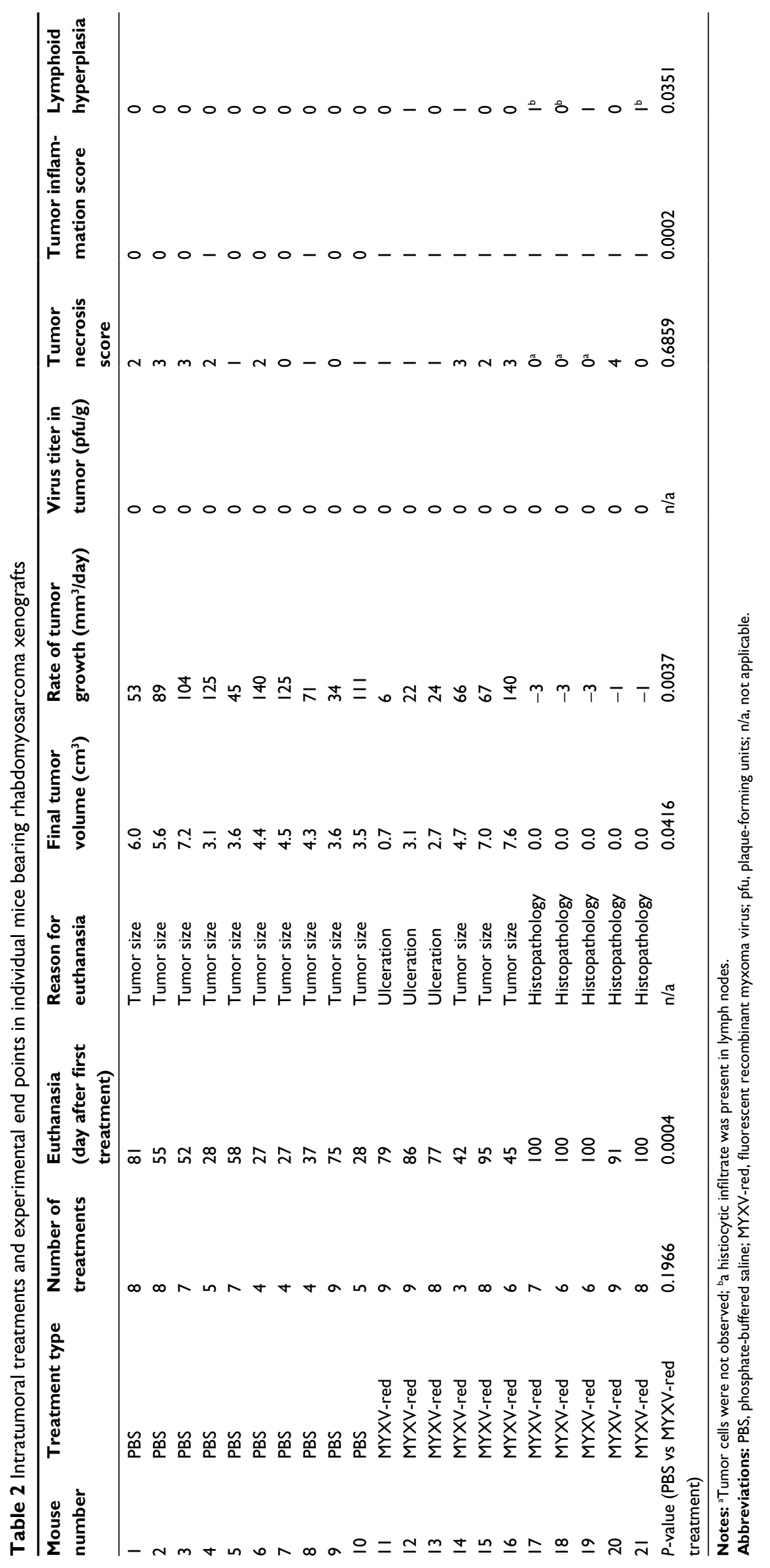




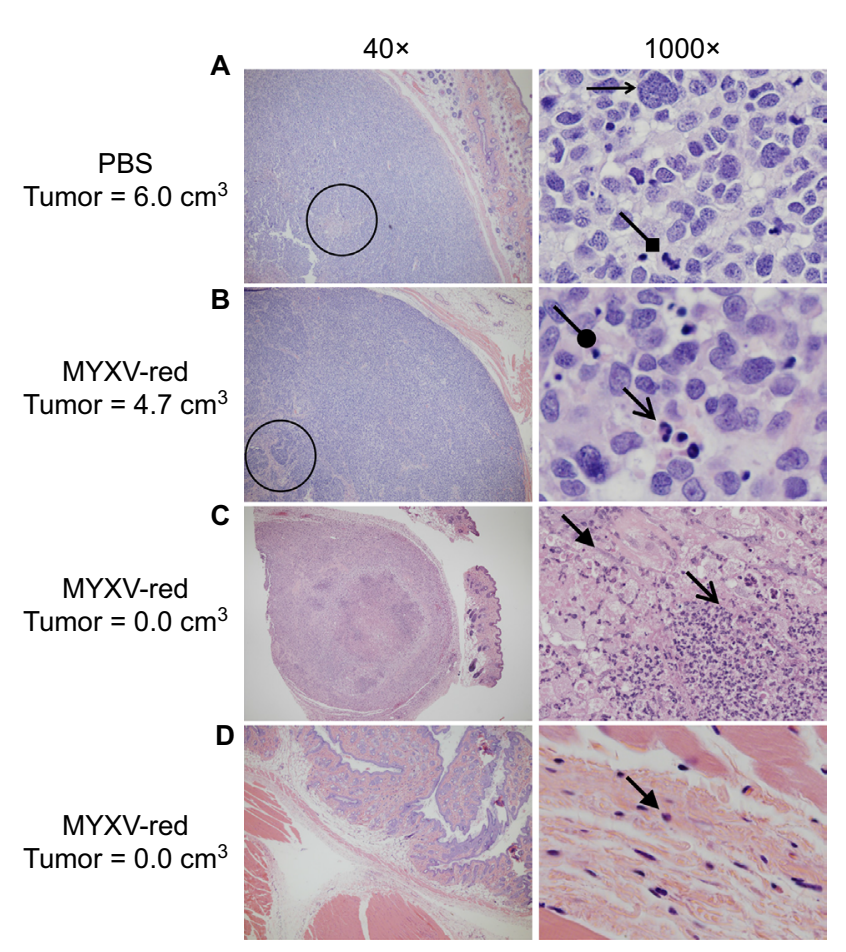

Figure 3 Tumor histopathology (H\&E).

Notes: Representative images of tissue from euthanized mice at the site of rhabdomyosarcoma xenograft implantation are shown. Magnification is listed above the images. Palpable, measurable tumors from (A) PBS- and (B) MYXV-redtreated mice appeared similar on histology. Areas of necrosis are indicated by the circles. Tumor cells are large with variably sized oval to irregular nuclei and stippled chromatin. Characteristics of malignancy include multinucleation (horizontal, small, open-ended arrow) and mitotic figures (diamond-shaped arrow). Dying cells with pyknotic nuclei (rounded arrow) and inflammatory cells including neutrophils (open arrow) are indicated. (C and D) Representative images of histopathology from mice that were treated with MYXV-red and did not have measurable tumors at euthanasia are shown. (C) One mouse had a subcutaneous focal area of necrosis, which contained many large vacuolated macrophages (closed arrow) and neutrophils (open arrow), and rare neoplastic cells (not shown). (D) No neoplastic cells were observed in other MYXV-red-treated mice that lacked microscopic tumors, but low numbers of inflammatory cells were noted (closed arrow).

Abbreviations: H\&E, hematoxylin and eosin; PBS, phosphate-buffered saline; MYXV-red, myxoma virus with red fluorescent protein.

treated with MYXV-red was similar to that of the PBS-treated masses although inflammation was observed in all MYXVred-treated tumors (Figure 3B). Inflammation was mild and mixed (neutrophils and mononuclear cells) in ten of eleven MYXV-red-treated mice but was severe and predominantly neutrophilic in one mouse with marked tumor necrosis (Figure 3C). Five of eleven mice treated with MYXV-red did not have a visible tumor when they were euthanized. Two of these mice had microscopic subcutaneous lesions at the site of virus inoculation that were composed of low numbers of neoplastic cells and showed mild inflammation; marked necrosis also was observed in the lesion from one of these mice. Neoplastic cells were not found in three of the mice, but mild inflammation was observed in the subcutaneous area where the tumor was present (Figure 3D). The presence of inflammation within the area of the tumor was significantly increased in MYXV-red-treated mice ( $P$-value 0.0002$)$. Key data for individual mice and the statistical significance of combined data from MYXV-treated mice compared to PBStreated mice are reported in Table 2.

\section{Virus was not detected in internal organs or RMS tumors}

To determine if MYXV-red was replicating within the tumor microenvironment or internal organs, RK13 cells were inoculated with tissue homogenates and DNA was isolated from tissue homogenates for PCR analysis using three sets of primers designed to detect MYXV DNA. MYXV-red could not be detected at the site of CCL-136 cell injection or in any internal organs of any of the ten mice injected with PBS or eleven mice treated with MYXV-red. Viral titer data from the tissue homogenates are presented in Table 2 and Table S1. Representative images from PCR analysis are shown in Figure S1.

\section{Discussion}

While many viruses have been tested for use as oncolytic viruses, this is the first report of using a poxvirus as a potential virotherapeutic agent in an established RMS cancer model. Positive treatment effects were noted in eight of eleven MYXV-red-treated mice bearing RMS tumors. Tumor regression was documented in five of these mice, as indicated by negative tumor growth rates.

It is unlikely that tumor regression was caused solely by MYXV-red oncolysis; necrosis was observed in both PBStreated and MYXV-red-treated tumors. The presence of necrosis in large, expansive tumors (such as those observed in the PBS-treated mice) is common and is often caused by the lack of an adequate blood supply to the cells at the center of mass. ${ }^{32}$ A critical histologic difference between PBS and MYXV-red-treated mice in this study was the presence of inflammation within MYXV-red-treated tumors. Nude mice are athymic and lack T-cells, but have an intact innate immune system and produce functional neutrophils, histiocytic cells, natural killer cells, and developed (but not fully differentiated and activated) B-cells. Induction of an inflammatory response to the virus likely contributed to the decreased rate of tumor growth in MYXV-red-treated mice. Additionally, virus was not isolated from tumors of MYXV-red-treated mice at the time of euthanasia. This is in agreement with previous studies that have suggested the innate immune response enables mice to eliminate MYXV-red from tumors within 4-7 days of MYXV-red inoculation. ${ }^{21}$ We conclude that both oncolysis and inflammation likely contributed to the reduction of RMS growth rate in MYXV-red-treated mice.

MYXV-red proved to be an effective treatment in this xenograft model of RMS, but the antitumor effects of 
MYXV-red may be further improved using a recombinant MYXV that enhances cell death and/or expresses proinflammatory cytokines that recruit immune cells to the tumor bed. Deletion of a MYXV gene that encodes an antiapoptotic protein has been shown to enhance cell death in cell culture. ${ }^{13}$ It is unclear if this will improve oncolytic effects in vivo, or if recombinant MYXV lacking antiapoptotic proteins will be cleared from the tumor before effective viral replication and immunostimulation can occur. Interestingly, recombinant MYXV that encodes an interleukin-15 fusion protein increases natural killer and $\mathrm{CD} 8^{+} \mathrm{T}$-cell infiltrates and median survival time in immunocompetent mice with melanoma allografts. ${ }^{29}$ Analysis of further genetic improvements to this recombinant virus is actively being pursued.

Increased cell death and infiltration of cytotoxic cells induced by oncolytic viruses may enhance antitumor effects, but there is mounting evidence that necrotic cell death and the inflammation often induced by necrosis are key components of effective oncolytic virus treatment. ${ }^{33-35}$ Expression of granulocyte-monocyte colony stimulating factor, which recruits neutrophils and macrophages to the tumor bed, improves the outcome of several oncolytic viruses, including recombinant poxviruses. ${ }^{36-40}$ The importance of neutrophils in modulating the immune response is beginning to be realized, but their contribution to antitumor immunity is controversial. Neutrophil expression of pattern recognition receptors, release of $\mathrm{T}_{\mathrm{H}}$ 1-promoting cytokines, and cross-presentation of antigens affects innate and adaptive immune responses and may enhance antitumor effects of oncolytic viruses. However, neutrophil release of angiogenic factors, including vascular endothelial growth factor and matrix metalloproteinase 9, may enhance the viability of tumor cells. ${ }^{41}$

Extensive evaluation of intratumoral molecular changes associated with neutrophilic inflammation during proinflammatory oncolytic viral therapy is needed to determine if the benefits of neutrophilic inflammation outweigh the potential detrimental effects. Further studies are planned to assess the therapeutic effects of proinflammatory MYXV recombinants in additional RMS tumor models. Although the oncolytic effects of MYXV may be able to be enhanced, the data presented in this manuscript show that MYXV-red injections greatly improve the outcome in a RMS xenograft model and give hope that, in the near future, oncolytic virotherapy will benefit patients afflicted with RMS.

\section{Acknowledgments}

An anonymous donor provided the majority of funding for this project. Additional funding was provided by Colorado State University, Fort Collins, CO, USA.

\section{Disclosure}

The authors report no conflicts of interest in this work.

\section{References}

1. Casanova M, Meazza C, Favini F, Fiore M, Morosi C, Ferrari A. Rhabdomyosarcoma of the extremities: a focus on tumors arising in the hand and foot. Pediatr Hematol Oncol. 2009;26(5):321-331.

2. Fulda S. Targeting apoptosis resistance in rhabdomyosarcoma. Curr Cancer Drug Targets. 2008;8(6):536-544.

3. Cinatl Jr J, Cinatl J, Michaelis M, et al. Potent oncolytic activity of multimutated herpes simplex virus $\mathrm{G} 207$ in combination with vincristine against human rhabdomyosarcoma. Cancer Res. 2003;63(7):1508-1514.

4. Pol J, Kroemer G, Galluzzi L. First oncolytic virus approved for melanoma immunotherapy. Oncoimmunology. 2015;5(1):e1115641.

5. Andrewes $\mathrm{CH}$, Harisijades S. Propagation of myxoma virus in one-day old mice. Br J Exp Pathol. 1955;36(1):18-21.

6. Fenner F, Woodroffe GM. The pathogenesis of infectious myxomatosis: the mechanism of infection and the immunological response in the European rabbit (Oryctolagus cuniculus). Br J Exp Pathol. 1953;34: 400-411.

7. Fenner F. Adventures with poxviruses of vertebrates. FEMS Microbiol Rev. 2000;24:123-133.

8. Gorski J, Mizak B, Chrobocinska M. Control of rabbit myxomatosis in Poland. Rev Sci Tech. 1994;13(3):869-879.

9. Jackson EW, Dorn CR, Saito JK, McKercher DG. Absence of serological evidence of myxoma virus infection in humans exposed during an outbreak of myxomatosis. Nature. 1966;211:313-314.

10. McCabe VJ, Tarpey I, Spibey N. Vaccination of cats with an attenuated recombinant myxoma virus expressing feline calicivirus capsid protein. Vaccine. 2002;20(19):2454-2462.

11. McCabe VJ, Spibey N. Potential for broad-spectrum protection against feline calicivirus using an attenuated myxoma virus expressing a chimeric FCV capsid protein. Vaccine. 2005;23(46-47):5380-5388.

12. Pignolet B, Boullier S, Gelfi J, et al. Safety and immunogenicity of myxoma virus as a new viral vector for small ruminants. J Gen Virol. 2008;89(Pt 6): 1371-1379.

13. Urbasic AS, Hynes S, Somrak A, et al. Oncolysis of canine tumor cells by myxoma virus lacking the serp2 gene. Am J Vet Res. 2012;73(8): $1252-1261$.

14. MacNeill AL, Moldenhauer T, Doty R, Mann T. Myxoma virus induces apoptosis in cultured feline carcinoma cells. Res Vet Sci. 2012;93(2): $1036-1038$.

15. Sypula J, Wang F, Ma Y, Bell J, McFadden G. Myxoma virus tropism in human tumor cells. Gene Ther Mol Biol. 2004;8:103-114.

16. Woo Y, Kelly KJ, Stanford MM, et al. Myxoma virus is oncolytic for human pancreatic adenocarcinoma cells. Ann Surg Oncol. 2008;15(8): 2329-2335.

17. Wang F, Ma Y, Barrett JW, et al. Disruption of Erk-dependent type I interferon induction breaks the myxoma virus species barrier. Nat Immunol. 2004;5(1):1266-1274.

18. Wang G, Barrett JW, Stanford M, et al. Infection of human cancer cells with myxoma virus requires Akt activation via interaction with a viral ankyrin-repeat host range factor. Proc Natl Acad Sci USA. 2006;103(12): $4640-4645$.

19. Stanford MM, Shaban M, Barrett JW, et al. Myxoma virus oncolysis of primary and metastatic B16F10 mouse tumors in vivo. Mol Ther. 2008; 16(1):52-59.

20. Thomas DL, Doty R, Tosic V, et al. Myxoma virus combined with rapamycin treatment enhances adoptive $\mathrm{T}$ cell therapy for murine melanoma brain tumors. Cancer Immunol Immunother. 2011;60(10): 1461-1472.

21. Doty RA, McFadden G, Roy EJ, MacNeill AL. Histological evaluation of intratumoral myxoma virus treatment in an immunocompetent mouse model of melanoma. Oncolytic Virother. 2013;2:1-17.

22. Liu J, Wennier S, Reinhard M, Roy E, MacNeill A, McFadden G. Myxoma virus expressing interleukin-15 fails to cause lethal myxomatosis in European rabbits. J Virol. 2009;83(11):5933-5938. 
23. MacNeill AL, Turner PC, Moyer RW. Mutation of the myxoma virus SERP2 P1-site to prevent proteinase inhibition causes apoptosis in cultured RK-13 cells and attenuates disease in rabbits, but mutation to alter specificity causes apoptosis without reducing virulence. Virology. 2006;356(1-2):12-22.

24. Everett H, Barry M, Sun XJ, et al. The myxoma poxvirus protein, M11L, prevents apoptosis by direct interaction with the mitochondrial permeability transition pore. J Exp Med. 2002;196(9):1127-1139.

25. Everett H, McFadden G. Poxviruses and apoptosis: a time to die. Curr Opin Microbiol. 2002;5(4):395-402.

26. Lun X, Yang W, Alain T, et al. Myxoma virus is a novel oncolytic virus with significant antitumor activity against experimental human gliomas. Cancer Res. 2005;65(2):9982-9990.

27. Lun X, Alain T, Zemp FJ, et al. Myxoma virus virotherapy for glioma in immunocompetent animal models: optimizing administration routes and synergy with rapamycin. Cancer Res. 2010;70(2):598-608.

28. Lun XQ, Zhou H, Alain T, et al. Targeting human medulloblastoma: oncolytic virotherapy with myxoma virus is enhanced by rapamycin. Cancer Res. 2007;67(18):8818-8827.

29. Tosic V, Thomas DL, Kranz DM, et al. Myxoma virus expressing a fusion protein of interleukin-15 (IL15) and IL15 receptor alpha has enhanced antitumor activity. PLoS One. 2014;9(10):e109801.

30. Marampon F, Bossi G, Ciccarelli C, et al. MEK/ERK inhibitor U0126 affects in vitro and in vivo growth of embryonal rhabdomyosarcoma. Mol Cancer Ther. 2009;8(3):543-551.

31. Tomayko MM, Reynolds CP. Determination of subcutaneous tumor size in athymic (nude) mice. Cancer Chemother Pharmacol. 1989;24(3):148-154.

32. Cullen JM, Page R, Misdorp W. An overview of cancer pathogenesis, diagnosis, and management. In: Meuten DJ, editor. Tumors in Domestic Animals. Vol 4. Ames, IA: Blackwell Publishing; 2002:13.
33. Grote D, Cattaneo R, Fielding AK. Neutrophils contribute to the measles virus-induced antitumor effect : enhancement by granulocyte macrophage colony-stimulating factor expression neutrophils contribute to the measles virus-induced antitumor effect : enhancement by granulocyte macrophage. Cancer Res. 2003;63:6463-6468.

34. Hwang T-HH, Moon A, Burke J, et al. A mechanistic proof-of-concept clinical trial with JX-594, a targeted multi-mechanistic oncolytic poxvirus, in patients with metastatic melanoma. Mol Ther. 2011; 19(10):1913-1922.

35. Wu L, Huang TG, Meseck M, et al. rVSV(M Delta 51)-M3 is an effective and safe oncolytic virus for cancer therapy. Hum Gene Ther. 2008;19(6): 635-647.

36. Fournier P, Aigner M, Schirrmacher V. Targeting of IL-2 and GM-CSF immunocytokines to a tumor vaccine leads to increased anti-tumor activity. Int J Oncol. 2011;38(6):1719-1729.

37. Lee JH, Roh MS, Lee YK, et al. Oncolytic and immunostimulatory efficacy of a targeted oncolytic poxvirus expressing human GM-CSF following intravenous administration in a rabbit tumor model. Cancer Gene Ther. 2010;17(2):73-79.

38. Lun X, Chan J, Zhou H, et al. Efficacy and safety/toxicity study of recombinant vaccinia virus JX-594 in two immunocompetent animal models of glioma. Mol Ther. 2010;18(11):1927-1936.

39. Malhotra S, Kim T, Zager J, et al. Use of an oncolytic virus secreting GM-CSF as combined oncolytic and immunotherapy for treatment of colorectal and hepatic adenocarcinomas. Surgery. 2007;141(4):520-529.

40. Parviainen S, Autio K, Koskela M, et al. Incomplete but infectious vaccinia virions are produced in the absence of oncolysis in feline SCCF1 cells. PLoS One. 2015;10(3):1-12.

41. Mantovani A, Cassatella MA, Costantini C, Jaillon S. Neutrophils in the activation and regulation of innate and adaptive immunity. Nat Rev. 2011;11(8):519-531. 


\section{Supplementary materials}

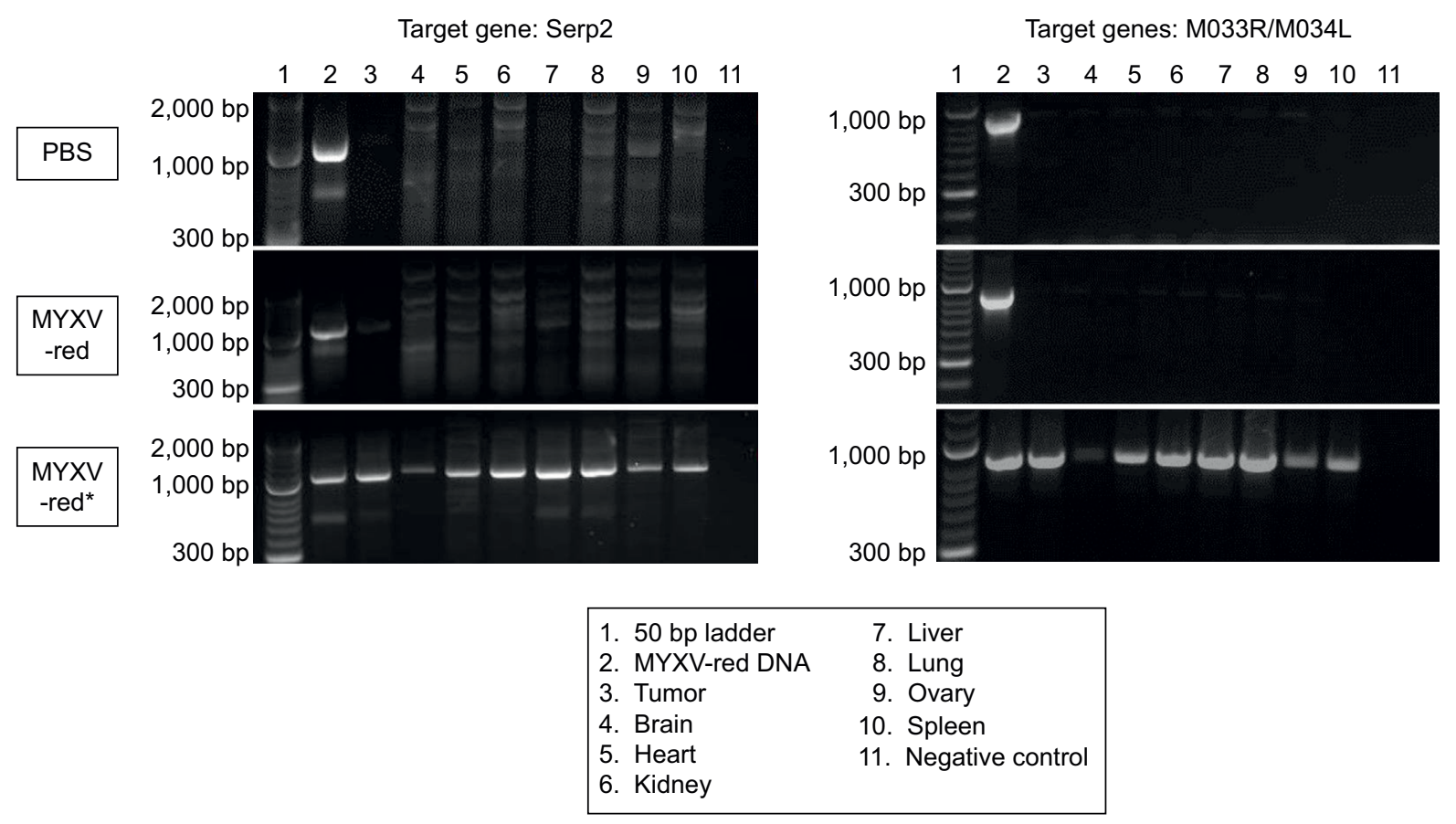

Figure SI Agarose gel electrophoresis of PCR products from two of the three primer sets designed to detect MYXV DNA are shown.

Notes: All tissue homogenates collected at euthanasia from PBS- and MYXV-red-treated mice were negative for MYXV DNA. However, MYXV DNA could be detected in tissue homogenates from MYXV-red*-treated mice that had an adverse reaction to treatment injection. ${ }^{*}$ This preparation of MYXW-red caused respiratory distress in five mice.

Abbreviations: PCR, polymerase chain reaction; PBS, phosphate-buffered saline; MYXV-red, myxoma virus with red fluorescent protein

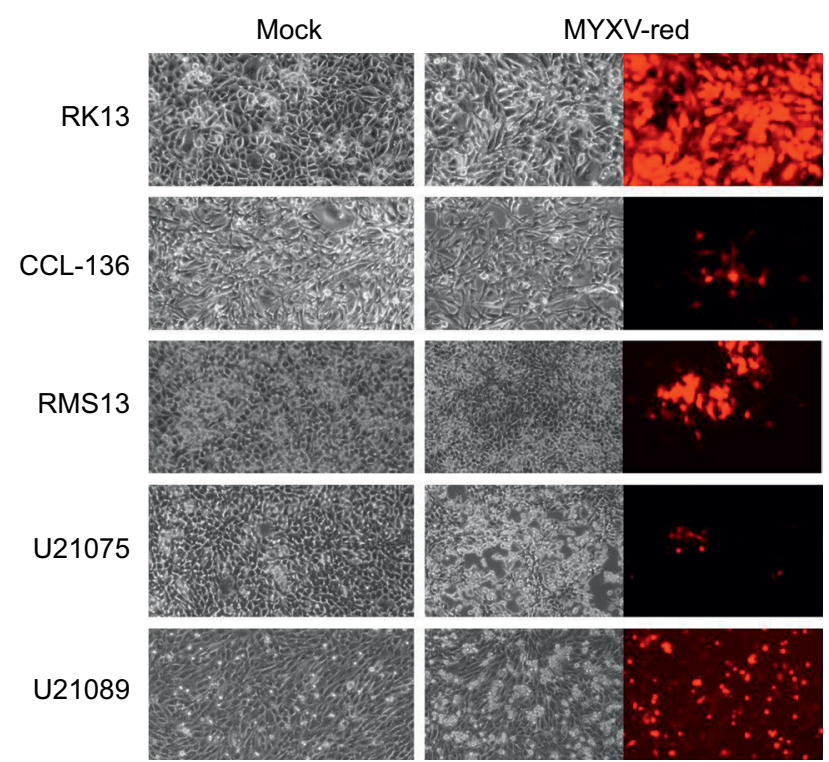

Figure S2 Cytopathic effects of incubating RMS cell cultures with MYXV-red (multiplicity of infection $=0.1$ ).

Notes: Forty-eight hours after inoculation with MYXV-red, red fluorescence is observed within RMS cells. Magnification 1000x.

Abbreviations: RMS, rhabdomyosarcoma; MYXV-red, myxoma virus with red fluorescent protein; RKI3, rabbit kidney epithelial cells; CCL-136, human rhabdomycosarcoma cells. 


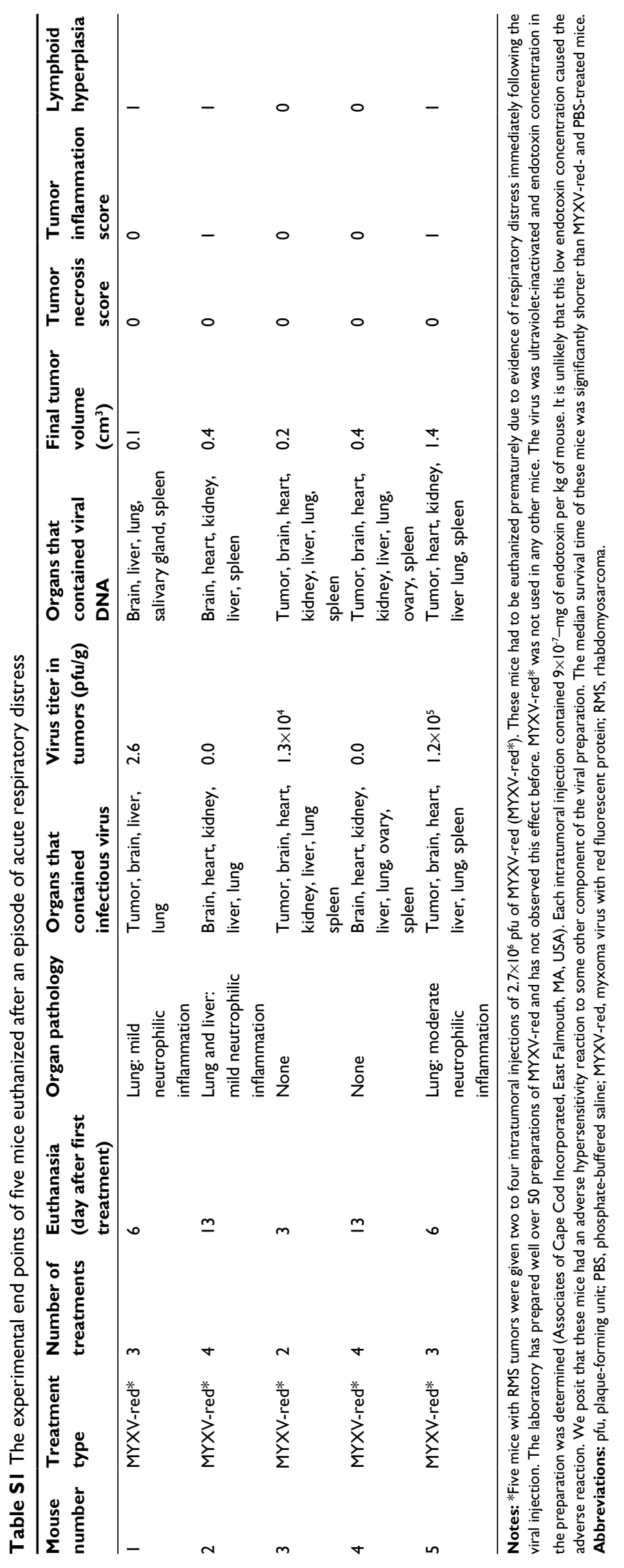


Oncolytic Virotherapy is an international, peer-reviewed, open access online journal publishing original research, study protocols, reviews, editorials and commentaries on all aspects of oncolytic virology, namely the application of oncolytic viruses for the treatment of cancer. Specific topics in the journal include: Rationale and theoretical aspects of oncolytic virotherapy including in vitro, in vivo and mathematical

Submit your manuscript here: http://www.dovepress.com/oncolytic-virotherapy-journal
Dovepress

modeling; and practical application and problem solving in the clinic including identification of potential responders through biomarkers and genetic profiling. The manuscript management system is completely online and includes a very quick and fair peer-review system, which is all easy to use. Visit http://www.dovepress.com/ testimonials.php to read real quotes from published authors. 